\begin{tabular}{|c|c|}
\hline Title & K-means clustering for support construction in diffractive imaging \\
\hline Author(s) & Hattanda, Shunsuke; Shioya, Hiroyuki; Maehara, Y osuke; Gohara, Kazutoshi \\
\hline Citation & $\begin{array}{l}\text { Journal of the Optical Society of A merica A, 31(3), } 470 \\
\text { https://doi.org/10.1364/JOSA A } 31.000470\end{array}$ \\
\hline Issue Date & $2014-02-05$ \\
\hline Doc URL & http:/hdl. handle.net/2115/54554 \\
\hline Type & article \\
\hline File Information & 2014_S.Hattanda_JOSA A .pdf \\
\hline
\end{tabular}

Instructions for use 


\title{
K-means clustering for support construction in diffractive imaging
}

\author{
Shunsuke Hattanda, ${ }^{1}$ Hiroyuki Shioya, ${ }^{2, *}$ Yosuke Maehara, ${ }^{3}$ and Kazutoshi Gohara ${ }^{3}$ \\ ${ }^{1}$ Division of Information and Systems Engineering, Muroran Institute of Technology, \\ 27-1 Mizumoto, Muroran 050-8585, Japan \\ ${ }^{2}$ College of Information and Systems, Muroran Institute of Technology, 27-1 Mizumoto, Muroran 050-8585, Japan \\ ${ }^{3}$ Division of Applied Physics, Graduate School of Engineering, Hokkaido University, N13, \\ W8, Kita-ku, Sapporo 060-8628, Japan \\ *Corresponding author: shioya@mmm.muroran-it.ac.jp
}

Received October 9, 2013; revised November 27, 2013; accepted December 6, 2013; posted December 17, 2013 (Doc. ID 198982); published February 5, 2014

\begin{abstract}
A method for constructing an object support based on K-means clustering of the object-intensity distribution is newly presented in diffractive imaging. This releases the adjustment of unknown parameters in the support construction, and it is well incorporated with the Gerchberg and Saxton diagram. A simple numerical simulation reveals that the proposed method is effective for dynamically constructing the support without an initial prior support. (c) 2014 Optical Society of America
\end{abstract}

OCIS codes: (100.5070) Phase retrieval; (100.3008) Image recognition, algorithms and filters.

http://dx.doi.org/10.1364/JOSAA.31.000470

\section{INTRODUCTION}

Diffractive imaging is used to obtain a target object image by reconstructing the missing phase in a Fourier domain, while the amplitude is observed. Two decades after Sayre's comments on the sampling theorem of Shannon [1], an iterative algorithm using the Fourier transforms for phase retrieval was presented by Gerchberg and Saxton [2]. The fundamental reason why phases can be retrieved from the oversampled diffraction intensities has been explained [3] . Diffractive imaging is widely spread over the field, requiring the reconstruction of the lost phase information using intensity measurements. Imaging using a soft x-ray diffraction pattern was first presented by Miao et al. [4]. Later, many related experiments were conducted using different sources, such as x rays [5-8], electron microscopes [ $\underline{9}-\underline{12}$ ], and tabletop light sources of lasers [13]. The diffractive imaging findings of these related works are summarized in [14]. In addition, theoretical and empirical analyses of phase retrieval have been performed in order to clarify the uniqueness and convergence of the widely used algorithms. The fundamental question of phase retrieval was raised in the early days of diffractive imaging research [15], and various theoretical, empirical, and experimental solutions of this problem have been investigated [16-24].

The object support is indispensable to obtain a phaseretrieved image under the Gerchberg and Saxton diagram. The tight support not generally given is desired to obtain a better result. The relationship between the convex properties of a target object and the support has been investigated in order to obtain a support by using the observed intensities [25-28]. On the other hand, the dynamical support extracting the object region in the phase-retrieval process has also been studied. The shrinking support using a Gaussian with a settled width was introduced [29], one of the advance settings of the object-domain constraint was presented by Oszlanyi and Suto
[30], and their algorithm was alternately used with the hybrid input-output (HIO) algorithm to yield a stable phase-retrieval process [31].

However, an important difficulty remains in terms of the dynamical object support. That is, there is no general rule for determining the unknown parameters of the region inherent to the shrinking and flipping method [29,30]. Providing an object support is important to obtain a plausible phaseretrieved object. Therefore, an appropriate method for providing an object support has been required in diffractive imaging.

In this paper, we present a novel method for determining an object support using the data-clustering method, K-means [32], in order to release the nuisance adjustment of unknown parameters in the support construction, and then the simultaneous usage with ER and HIO in the framework of the Gerchberg and Saxton diagram is established with a numerical example.

\section{PHASE RETRIEVAL}

The reconstruction of the Fourier phase using intensity measurements is presented in the cyclic transform of the Gerchberg-Saxton iterative algorithm [2] shown in Fig. 1. The prior object $\rho$ is transformed into $F$ by the Fourier transform, $F$ is replaced by $F^{\prime}$ (the amplitude is given by the experiment in the Fourier domain, and the phase of $F^{\prime}$ is the same as that of $F$, where the replaced amplitude is the constraint in the Fourier domain), $\rho^{\prime}$ is obtained by the inverse Fourier transform of $F^{\prime}$, and $\rho^{\prime}$ is replaced by the updated object as the next $\rho$ using the object-domain constraints. The object domain $X$ is defined as a discrete squared array, and the Fourier domain $K$ is also defined in the same way as domain $X$ with the discrete Fourier transform for practical computation.

The error reduction algorithm is a method of updating from the $n$th object $\rho_{n}$ to the $(n+1)$ th object $\rho_{n+1}$ as 


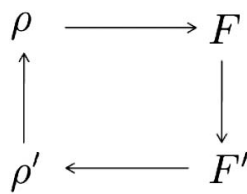

Fig. 1. Gerchberg and Saxton's iterative diagram for phase retrieval.

$$
\rho_{n+1}(\boldsymbol{r})=\left\{\begin{array}{cc}
\rho_{n}^{\prime}(\boldsymbol{r}) & \boldsymbol{r} \notin D \\
0 & \boldsymbol{r} \in D
\end{array},\right.
$$

where $D$ is the set of points at which $\rho_{n}^{\prime}$ violates the objectdomain constraints. In the initial state of the phase-retrieval process, a prior object is very far from the desired object function; hence the HIO algorithm is often used as an improved version of the updating method with respect to the region breaking the object-domain constraints [25]:

$$
\rho_{n+1}(\boldsymbol{r})= \begin{cases}\rho_{n}^{\prime}(\boldsymbol{r}) & \boldsymbol{r} \notin D \\ \rho_{n}(\boldsymbol{r})-\beta \rho_{n}^{\prime}(\boldsymbol{r}) & \boldsymbol{r} \in D\end{cases}
$$

where $\beta$ is a positive constant. The HIO yields a typical change to the object $\rho_{n}^{\prime}$ on the region that does not satisfy the objectdomain constraints. Both of these algorithms have been used connectively. The object support needs to be appropriately given as when using the $\mathrm{HIO}$ and ER. The autocorrelation of a target object is presented by the inverse transform of the Fourier intensity of the object. Fienup focused on this relation in the case in which a target object has a convex support, and presented some results concerning the object support generated by the Fourier intensity [25,28]. The usefulness of the autocorrelation is followed for such limited objects, except for the incompleteness of the Fourier intensity, including the Poisson noise or data missing on the Fourier domain.

\section{SUPPORT CONSTRUCTION AND K-MEANS CLUSTERING}

The object support is a delimited region where a target object is located. The region of the phase-retrieved object and its complement on the object domain should have nonzero intensity and zero intensity, respectively. Such prior information is given as the object-domain constraint. The tightness of the object support improves the convergence in the phase-retrieval process. The convergence of the retrieval process deteriorates according to the degree of looseness of the object support. It is difficult to count on a strictly tight support for the phase retrieval of the experimental diffraction patterns.

The object domain is divided into two kinds of regions; one is the object support, and the other is its complement. When $\rho^{\prime}(\boldsymbol{r})$ is an insufficiently phase-retrieved object in the Gerchberg and Saxton diagram, it is not clear whether each $r$ is the object support or not, and such ambiguity is unavoidable without a tight object support. In order to confidently extract an object support, a process for discriminating between the zero intensity region and the nonzero intensity region must be established. We focus on the distribution of $\left|\rho^{\prime}\right|$, and newly introduce the K-means clustering [32,33] for dividing a set of $\left\{\left|\rho^{\prime}\left(\boldsymbol{r}_{1}\right)\right|, \cdots,\left|\rho^{\prime}\left(\boldsymbol{r}_{N}\right)\right|\right\}$ into two classes; one is the point of the object support, and the other is in the region not belonging to the support, where $N$ is the total pixel number of the object domain $X$.
Data clustering is a method used to divide a set of data into two or more subsets with respect to a certain similarity measure on the data space. The K-means algorithm is a representative data-clustering algorithm using a metric between the center point of a class and each data point [32-35]. Various data-clustering algorithms based on the K-means have been introduced and applied to data mining and analysis in the field of information science and engineering [33].

Given point set $S$ on a metric space, the K-means clustering algorithm is presented as follows. As an initial setting, all points are randomly divided into $k$ subsets, $C_{0}, C_{1}, \cdots, C_{k-1}$, where $C_{j} \neq \phi$ for $\forall j$ and $S=\cup_{j=0}^{k-1} C_{j}$. Each subset is called a "class," and its index is called a "label" of the point of the class. $c_{j}$ is the center by averaging all points of $C_{j}$ for $j=0, \cdots, k-1$; that is, $c_{j}=\sum_{p \in C_{j}} p / J$, where $J$ is the cardinality of $C_{j}$. Then a new label of point $q$ is given by the index of the center whose distance from the point is minimum; that is,

$$
\text { New label of } q=\arg \min _{j=0, \cdots, k-1} d\left(q, c_{j}\right) \text {, }
$$

where $d(\cdot, \cdot)$ is a distance between two points. The new labels of the point set are given by the nearest centers, and the class assignment is updated with these labels. The updating of the labels is iterated until these changes subside, and the data clustering is then finished.

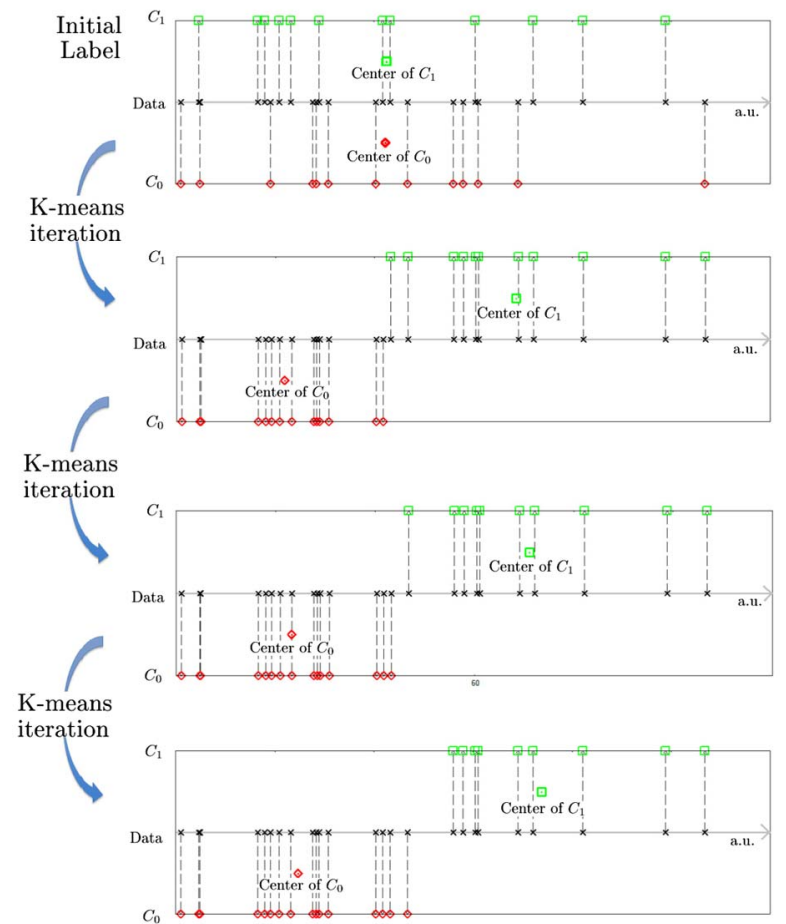

Fig. 2. Example of K-means clustering is presented for labeling a given data set with two classes, $C_{0}$ and $C_{1}$. The black " $\times$ " is a point of the data set, and its label is presented above and below the axis in green and red, respectively. An initial label for each data point is randomly given in the top figure, and the center of each class is indicated by averaging the points of the class. The iteration of the K-means clustering algorithm is performed to update the label by the nearest center from the point. The updating of the labels is iterated until these changes subside, and the data clustering is finished. 
A simple schematic figure for K-means clustering of a onedimensional data set with two classes is presented in Fig. 2. The data are shown in black on the horizontal axis of each figure. Two classes of $C_{0}$ and $C_{1}$ are assigned at below and above the axis, respectively. The points belonging to both classes $C_{0}$ and $C_{1}$ are presented as red and green, respectively. An initial label is randomly given, and the label of each point is updated by the K-means iteration, i.e., Eq. (3).

The dynamical object support by the K-means clustering is described as follows. Let $N$ be the cardinality of the object domain $X$, and $\rho^{\prime}$ the object obtained through the Fourierdomain constraints of the diagram in Fig. 1. We focus on the nonnegative real set $\left\{\left|\rho^{\prime}\left(\boldsymbol{r}_{1}\right)\right|, \cdots,\left|\rho^{\prime}\left(\boldsymbol{r}_{N}\right)\right|\right\}$, and introduce the K-means algorithm for classifying the set into two classes, $C_{0}$ and $C_{1}$. The centers of the classes are obtained by averaging all elements of each class. Let $C_{1}$ be a class whose center value is larger than that of another class. As a result, $C_{1}$ corresponds to the object support.

This method for giving the object support is effectively inserted into the Gerchberg and Saxton diagram. Figure 3 presents the diagram including the dynamical object support by the K-means clustering procedure. Our proposed method for extracting the object support is algorithmically described as follows.

Step 1: Obtain $\rho^{\prime}$ by the inverse Fourier transform in the Gerchberg-Saxton diagram.

Step 2: Give the initial labels for the point set $\left\{\left|\rho^{\prime}\left(\boldsymbol{r}_{1}\right)\right|, \cdots,\left|\rho^{\prime}\left(\boldsymbol{r}_{N}\right)\right|\right\}$.

Step 3: Generate two classes for the point set by Eq. (3).

Step 4: Iterate Step 3 until changing is complete for each point.

Step 5: Extract a class for whom the center is larger than that for another class.

Step 6: Obtain an object support, and return to the objectdomain constraints in the Gerchberg-Saxton diagram.

The computational cost of the K-means clustering is a linear order due to the object-domain size multiplied by the iteration number for updating the labels, and it is less than the cost of the fast Fourier transform.

\section{NUMERICAL EXAMPLE AND CONCLUSION}

The following is an example of our numerical simulations to present the validity of the K-means clustering for dynamical object support in diffractive imaging. We choose a twodimensional figure $\rho_{\text {org }}$ as the original object on the discrete square array domain $X(256 \times 256)$ shown as Fig. $\underline{4(a) .} F_{\text {org }}$ is the Fourier intensity of $\rho_{\text {org }}$ shown as Fig. $\underline{4(b)}$. Figure $\underline{4(\mathrm{c})}$

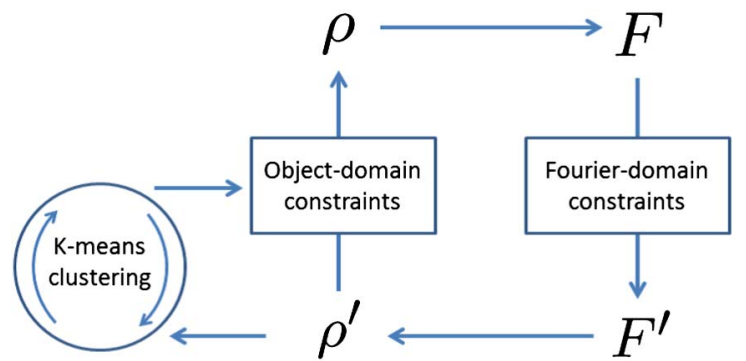

Fig. 3. K-means clustering is inserted into the Gerchberg and Saxton iterative diagram for the dynamical construction of object support.

is the Fourier intensity contaminated by Poisson noise using a random sample from the Poisson distribution with the intensity $I_{\text {org }}=\left|F_{\text {org }}\right|^{2}$ as the expectation.

The Poisson-noise-contaminated intensity for each element $\boldsymbol{k}$ in the Fourier domain $K$ is obtained by the following equation:

$$
\text { Poisson }\left\{c I_{\text {org }}(\boldsymbol{k})\right\} \sim I_{\text {noise }}(\boldsymbol{k})
$$

where $c$ is a coefficient based on $c=$ (total count)/ $\sum_{k \in K} I_{\text {org }}(k)$ and the total count is settled by $10^{5}$, and “ " means that the right part of the equation is a random sample from the probability distribution of the left part [24]. The common setting of the simulation is as follows. The $\mathrm{HIO}$ (10 iterations) and ER (10 iterations) are reciprocally used. The real positive condition for the target object is used. The left and right figures of Fig. $\underline{4(\mathrm{~d})}$ are a loose support (the oversampling ratio is 3.62 ) and a phase-retrieved object using the support, respectively. The left and right figures of Fig. $\underline{4(\mathrm{e})}$ are the strictly tight support and a phase-retrieved object using the support, respectively. The left and right figures of Fig. 4(f) are the perfect support and a phase-retrieved object using the support, respectively.

Figure $4(\mathrm{~g})$ is a phase-retrieved object obtained using the dynamical support by the shrinking method [29]. Every 20 iterations, the HIO (10 iterations) and ER (10 iterations),
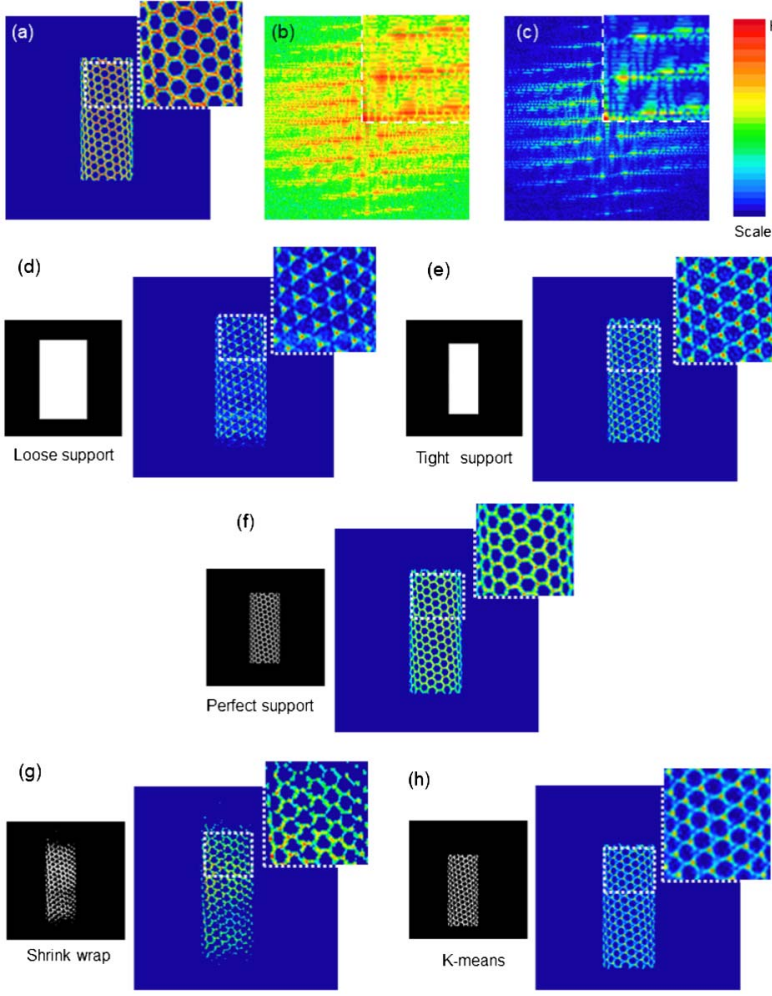

Fig. 4. (a) Original image. (b) Fourier intensity of (a). (c) Fourier intensity of (a) contaminated by Poisson noise with a total count of $10^{5}$. (d) Retrieved image (right) using a loose-object support whose oversampling ratio is 3.62 (left). (e) Retrieved image (right) using a strictly tight support (left). (f) Retrieved image (right) using the perfect support (left). (g) Image obtained by the shrink-wrap algorithm (right), and the obtained object support (left). (h) Image obtained by our algorithm periodically using K-means clustering (right), and the obtained object support (left). The upper right of each figure is a magnification of the area indicated by the white broken line. 


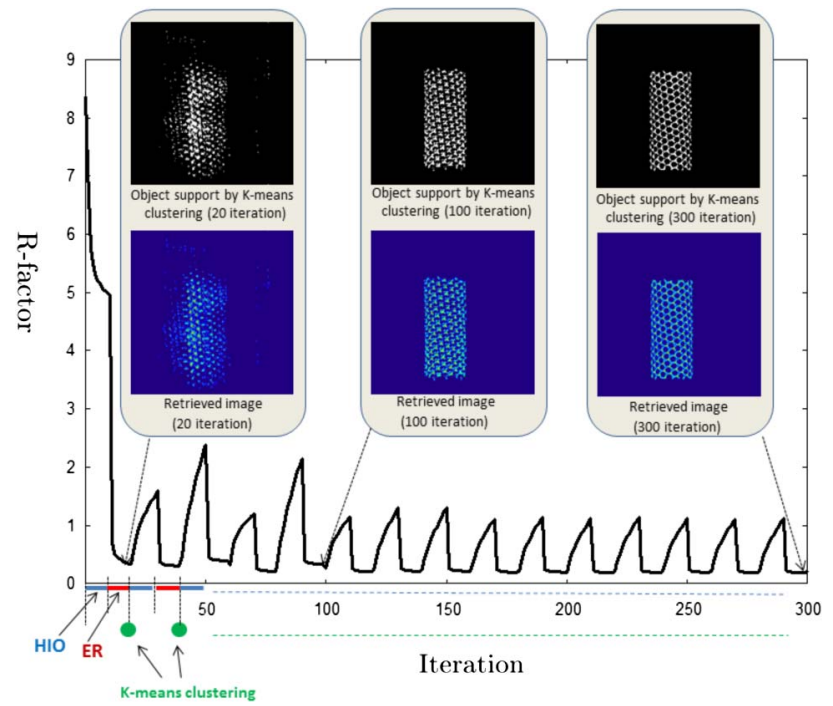

Fig. 5. Object supports were obtained by the K-means extraction, and the corresponding retrieved images are presented with the R-factor. 10 iterations are assigned for the HIO and ER algorithms, respectively, and both are alternately assigned. The K-means for extracting the object support is introduced after every 20 iterations of the HIO and ER algorithm.

we convolve the reconstructed image with a Gaussian of width $\sigma$ to find the new support mask. The mask is obtained by applying a threshold at $20 \%$ of the maximum-absolute value in the image. The width $\sigma$ is set to 3 pixels in the first iteration, and is reduced by $1 \%$ every 20 iterations. The left panel of Fig. $\underline{4(\mathrm{~g})}$ is the object support obtained by the shrinking method. All the reconstructed objects are obtained by a sufficient number of iterations on the Gerchberg-Saxton diagram.

Next, we consider a case using the dynamical object support by the K-means clustering method. The iteration numbers of the HIO and ER are the same as in the cases of the loose, tight, and perfect supports, and the shrinking method. Figure $4(\mathrm{~h})$ is an image obtained by periodically using K-means clustering for determining the object support for every 20 iterations of the Gerchberg-Saxton diagram. A randomly assigned label is used as an initial label at the start of each K-means clustering process. The left panel of Fig. $4(\mathrm{~h})$ is the object support obtained by the K-means clustering method. As a result, our method gives a successful case for determining the object support as a comparison of the shrinking method, and provides a plausible image in the same way as the case giving a tight object support. The obtained support is also plausible as a comparison of the perfect support. Figure 5 presents the changes of the R-factors, retrieved images, and corresponding object supports obtained by the K-means clustering.

The object support is one of the object-domain constraints, and the degree of looseness influences the plausibility of the phase-retrieved results. In this paper, K-means clustering for extracting the object support is first introduced, to the best of our knowledge. This releases the adjustment of the parameters of the object-support construction, and harmonizes the confidential framework of the Gerchberg and Saxton diagram. The method is used for extracting the object support of various objects, and should help to improve the spatial resolution in diffractive imaging.

\section{ACKNOWLEDGMENTS}

We thank the reviewers for their constructive comments and suggestions. This research was supported by Japan Grantin-Aid for Scientific Research (B) 24300065, and the Japan Science and Technology Agency (JST).

\section{REFERENCES}

1. D. Sayre, "Some implications of a theorem due to Shannon," Acta Crystallogr. 5, 843 (1952).

2. R. W. Gerchberg and W. O. Saxton, "A practical algorithm for the determination of phase from image and diffraction plane pictures," Optik 35, 237-246 (1972).

3. J. Miao, D. Sayre, and H. N. Chapman, "Phase retrieval from the magnitude of the Fourier transforms of nonperiodic objects," J. Opt. Soc. Am. A 15, 1662-1669 (1998).

4. J. Miao, P. Charalambous, J. Kirz, and D. Sayre, "Extending the methodology of X-ray crystallography to allow imaging of micrometre-sized non-crystalline specimens," Nature 400, 342-344 (1999).

5. J. Miao, T. Ishikawa, B. Johnson, E. H. Anderson, B. Lai, and K. O. Hodgson, "High resolution 3D x-ray diffraction microscopy," Phys. Rev. Lett. 89, 088303 (2002).

6. J. Miao, T. Ishikawa, E. H. Anderson, and K. O. Hodgson, "Phase retrieval of diffraction patterns from noncrystalline samples using the oversampling method," Phys. Rev. B 67, 174104 (2003).

7. Y. Nishino, J. Miao, and T. Ishikawa, "Image reconstruction of nanostructured nonperiodic objects only from oversampled hard x-ray diffraction intensities," Phys. Rev. B 68, 220101(R) (2003).

8. H. N. Chapman, A. Barty, M. J. Bogan, S. Boutet, M. Frank, S. P. Hau-Riege, S. Marchesini, B. W. Woods, S. Bajt, W. H. Benner, R. A. London, E. Plönjes, M. Kuhlmann, R. Treusch, S. Düsterer, T. Tschentscher, J. R. Schneider, E. Spiller, T. Möller, C. Bostedt, M. Hoener, D. A. Shapiro, K. O. Hodgson, D. van der Spoel, F. Burmeister, M. Bergh, C. Caleman, G. Huldt, M. M. Seibert, F. R. N. C. Maia, R. W. Lee, A. Szöke, N. Timneanu, and J. Hajdu, "Femtosecond diffractive imaging with a soft-x-ray free-electron laser," Nat. Phys. 2, 839-843 (2006).

9. U. Weierstall, Q. Chen, J. C. H. Spence, M. R. Howells, M. Isaacson, and R. R. Panepucci, "Image reconstruction from electron and X-ray diffraction patterns using iterative algorithms: experiment and simulation," Ultramicroscopy 90, 171-195 (2002).

10. J. M. Zuo, I. Vartanyants, M. Gao, R. Zhang, and L. A. Nagahara, "Atomic resolution imaging of a carbon nanotube from diffraction intensities," Science 300, 1419-1421 (2003).

11. O. Kamimura, K. Kawahara, T. Doi, T. Dobashi, T. Abe, and K Gohara, "Diffraction microscopy using $20 \mathrm{kV}$ electron beam for multiwall carbon nanotubes," Appl. Phys. Lett. 92, 024106 (2008).

12. S. Morishita, J. Yamasaki, K. Nakamura, T. Kato, and N. Tanaka, "Diffractive imaging of the dumbbell structure in silicon by spherical-aberation-corrected electron diffraction," Appl. Phys. Lett. 93, 183103 (2008).

13. R. L. Sandberg, A. Paul, D. A. Raymondson, S. Hädrich, D. M. Gaudiosi, J. Holtsnider, R. I. Tobey, O. Cohen, M. M. Murnane, and H. C. Kapteyn, "Lensless diffractive imaging using tabletop coherent high-harmonic soft-x-ray beams," Phys. Rev. Lett. 99 098103 (2007).

14. J. C. H. Spence, Science of Microscopy, P. W. Hawkes and J. C. H. Spence, eds. (Springer, 2007).

15. A. Walter, "The question of phase retrieval in optics," Opt. Acta 10, 41-49 (1963).

16. J. R. Fienup, "Phase retrieval algorithms: a comparison," Appl. Opt. 21, 2758-2769 (1982).

17. J. R. Fienup, "Reconstruction of an object from the modulus of its Fourier transform," Opt. Lett. 3, 27-29 (1978).

18. J. R. Fienup, "Space object imaging through the turbulent atmosphere," Opt. Eng. 18, 185529 (1979).

19. B. R. Frieden and D. G. Currie, "On unfolding the autocorrelation function," J. Opt. Soc. Am. 66, 1111 (1976). 
20. R. H. T. Bates, "Fourier phase problems are uniquely solvable in more than one dimension. I. Underlying theory," Optik 61, 247-262 (1982).

21. K. L. Garden and R. H. T. Bates, "Fourier phase problems are uniquely solvable in more than one dimension. II. One dimensional considerations," Optik 62, 131-142 (1982).

22. W. R. Fright and R. H. T. Bates, "Fourier phase problems are uniquely solvable in more than one dimension. III. Computational examples for two dimension," Optik 62, 219-230 (1982).

23. V. Elser, "Phase retrieval by iterated projections," J. Opt. Soc. Am. A 20, 40-55 (2003).

24. H. Shioya, Y. Maehara, and K. Gohara, "Spherical shell structure of distribution of images reconstructed by diffractive imaging," J. Opt. Soc. Am. A 27, 1214-1218 (2010).

25. J. R. Fienup, T. R. Crimmins, and W. Holsztynski, "Reconstruction of the support an object from the support of its autocorrelation," J. Opt. Soc. Am. A 72, 610-624 (1982).

26. J. R. Fienup, "Phase retrieval using boundary conditions," J. Opt. Soc. Am. A 3, 284-288 (1986).

27. J. R. Fienup and C. C. Wackerman, "Phase-retrieval stagnation problems and solutions," J. Opt. Soc. Am. A 3, 1897-1907 (1986).
28. T. R. Crimmins, J. R. Fienup, and B. J. Thelen, "Improved bounds on object support from autocorrelation support and application to phase retrieval," J. Opt. Soc. Am. A 7, 3-13 (1990).

29. S. Marchesini, H. He, H. N. Chapman, S. P. Hau-Riege, A. Noy, M. R. Howells, U. Weierstall, and J. C. H. Spence, "X-ray image reconstruction from a diffraction pattern alone," Phys. Rev. B 68, 140101 (2003).

30. G. Oszlanyi and A. Suto, "Ab initio structure solution by charge flipping," Acta Crystallogr. Sect. A 60, 134-141 (2004).

31. J. S. Wu, U. Weierstall, J. C. Spence, and C. T. Koch, "Iterative phase retrieval without support," Opt. Lett. 29, 2737-2739 (2004).

32. J. B. MacQueen, "Some methods for classification and analysis of multivariate observations," in Proceedings of 5th Berkeley Symposium on Mathematical Statistics and Probability (University of California, 1967), pp. 281-297.

33. A. K. Jain, N. M. Murty, and P. J. Flynn, "Data clustering: a review," ACM Comput. Surv. 31, 264-323 (1999).

34. J. A. Hartigan, Clustering Algorithms (Wiley, 1975).

35. J. A. Hartigan and M. A. Wong, "A K-means clustering algorithm,” J. R. Stat. Soc. Ser. C 28, 100-108 (1979). 